\title{
Inferior Limit, Superior Limit and Convergence of Sequences of Extended Real Numbers
}

\author{
Hiroshi Yamazaki \\ Shinshu University \\ Nagano, Japan
}

\author{
Yasunari Shidama \\ Shinshu University \\ Nagano, Japan
}

\author{
Noboru Endou \\ Gifu National College of Technology \\ Japan
}

\author{
Hiroyuki Okazaki \\ Shinshu University \\ Nagano, Japan
}

\begin{abstract}
Summary. In this article, we extended properties of sequences of real numbers to sequences of extended real numbers. We also introduced basic properties of the inferior limit, superior limit and convergence of sequences of extended real numbers.
\end{abstract}

MML identifier: RINFSUP2, version: 7.8.05 4.87.985

The notation and terminology used in this paper are introduced in the following articles: [18], [19], [1], [17], [20], [5], [21], [6], [7], [16], [2], [3], [8], [15], [13], [14], [12], [11], [22], [4], [10], and [9].

We adopt the following convention: $n, m, k$ are elements of $\mathbb{N}, X$ is a non empty subset of $\overline{\mathbb{R}}$, and $Y$ is a non empty subset of $\mathbb{R}$.

Next we state four propositions:

(1) If $X=Y$ and $Y$ is upper bounded, then $X$ is upper bounded and $\sup X=\sup Y$.

(2) If $X=Y$ and $X$ is upper bounded, then $Y$ is upper bounded and $\sup X=\sup Y$.

(3) If $X=Y$ and $Y$ is lower bounded, then $X$ is lower bounded and $\inf X=$ $\inf Y$.

(4) If $X=Y$ and $X$ is lower bounded, then $Y$ is lower bounded and $\inf X=$ $\inf Y$. 
Let $s_{1}$ be a sequence of extended reals. The functor $\sup s_{1}$ yields an element of $\overline{\mathbb{R}}$ and is defined by:

(Def. 1) $\sup s_{1}=\sup \operatorname{rng} s_{1}$.

The functor inf $s_{1}$ yields an element of $\overline{\mathbb{R}}$ and is defined as follows:

(Def. 2) $\inf s_{1}=\inf \operatorname{rng} s_{1}$.

Let $s_{1}$ be a sequence of extended reals. We say that $s_{1}$ is lower bounded if and only if:

(Def. 3) $\operatorname{rng} s_{1}$ is lower bounded.

We say that $s_{1}$ is upper bounded if and only if:

(Def. 4) $\quad \operatorname{rng} s_{1}$ is upper bounded.

Let $s_{1}$ be a sequence of extended reals. We say that $s_{1}$ is bounded if and only if:

(Def. 5) $s_{1}$ is upper bounded and lower bounded.

In the sequel $s_{1}$ is a sequence of extended reals.

One can prove the following proposition

(5) For all $s_{1}, n$ holds $\left\{s_{1}(k) ; k\right.$ ranges over elements of $\left.\mathbb{N}: n \leq k\right\}$ is a non empty subset of $\overline{\mathbb{R}}$.

Let $s_{1}$ be a sequence of extended reals. The inferior realsequence $s_{1}$ yields a sequence of extended reals and is defined by the condition (Def. 6).

(Def. 6) Let $n$ be an element of $\mathbb{N}$. Then there exists a non empty subset $Y$ of $\overline{\mathbb{R}}$ such that $Y=\left\{s_{1}(k) ; k\right.$ ranges over elements of $\left.\mathbb{N}: n \leq k\right\}$ and (the inferior realsequence $\left.s_{1}\right)(n)=\inf Y$.

Let $s_{1}$ be a sequence of extended reals. The superior realsequence $s_{1}$ yields a sequence of extended reals and is defined by the condition (Def. 7).

(Def. 7) Let $n$ be an element of $\mathbb{N}$. Then there exists a non empty subset $Y$ of $\overline{\mathbb{R}}$ such that $Y=\left\{s_{1}(k) ; k\right.$ ranges over elements of $\left.\mathbb{N}: n \leq k\right\}$ and (the superior realsequence $\left.s_{1}\right)(n)=\sup Y$.

We now state the proposition

(6) If $s_{1}$ is finite, then $s_{1}$ is a sequence of real numbers.

Let $f$ be a partial function from $\mathbb{N}$ to $\overline{\mathbb{R}}$. We say that $f$ is increasing if and only if:

(Def. 8) For all $m, n$ such that $m \in \operatorname{dom} f$ and $n \in \operatorname{dom} f$ and $m<n$ holds $f(m)<f(n)$.

We say that $f$ is decreasing if and only if:

(Def. 9) For all $m, n$ such that $m \in \operatorname{dom} f$ and $n \in \operatorname{dom} f$ and $m<n$ holds $f(m)>f(n)$.

We say that $f$ is non-decreasing if and only if: 
(Def. 10) For all $m, n$ such that $m \in \operatorname{dom} f$ and $n \in \operatorname{dom} f$ and $m \leq n$ holds $f(m) \leq f(n)$.

We say that $f$ is non-increasing if and only if:

(Def. 11) For all $m, n$ such that $m \in \operatorname{dom} f$ and $n \in \operatorname{dom} f$ and $m \leq n$ holds $f(m) \geq f(n)$.

One can prove the following two propositions:

(7)(i) $\quad s_{1}$ is increasing iff for all elements $n, m$ of $\mathbb{N}$ such that $m<n$ holds $s_{1}(m)<s_{1}(n)$,

(ii) $\quad s_{1}$ is decreasing iff for all elements $n, m$ of $\mathbb{N}$ such that $m<n$ holds $s_{1}(n)<s_{1}(m)$,

(iii) $\quad s_{1}$ is non-decreasing iff for all elements $n, m$ of $\mathbb{N}$ such that $m \leq n$ holds $s_{1}(m) \leq s_{1}(n)$, and

(iv) $\quad s_{1}$ is non-increasing iff for all elements $n, m$ of $\mathbb{N}$ such that $m \leq n$ holds $s_{1}(n) \leq s_{1}(m)$.

(8) (The inferior realsequence $\left.s_{1}\right)(n) \leq s_{1}(n)$ and $s_{1}(n) \leq$ (the superior realsequence $\left.s_{1}\right)(n)$.

Let us consider $s_{1}$. Observe that the superior realsequence $s_{1}$ is nonincreasing and the inferior realsequence $s_{1}$ is non-decreasing.

Let $s_{1}$ be a sequence of extended reals. The functor $\lim \sup s_{1}$ yields an element of $\overline{\mathbb{R}}$ and is defined by:

(Def. 12) $\lim \sup s_{1}=\inf \left(\right.$ the superior realsequence $s_{1}$ ).

The functor $\lim \inf s_{1}$ yields an element of $\overline{\mathbb{R}}$ and is defined by:

(Def. 13) $\liminf s_{1}=\sup \left(\right.$ the inferior realsequence $s_{1}$ ).

In the sequel $r_{1}$ is a sequence of real numbers.

The following propositions are true:

(9) If $s_{1}=r_{1}$ and $r_{1}$ is bounded, then the superior realsequence $s_{1}=$ the superior realsequence $r_{1}$ and $\lim \sup s_{1}=\lim \sup r_{1}$.

(10) If $s_{1}=r_{1}$ and $r_{1}$ is bounded, then the inferior realsequence $s_{1}=$ the inferior realsequence $r_{1}$ and $\liminf s_{1}=\liminf r_{1}$.

(11) If $s_{1}$ is bounded, then $s_{1}$ is a sequence of real numbers.

(12) If $s_{1}=r_{1}$, then $s_{1}$ is upper bounded iff $r_{1}$ is upper bounded.

(13) If $s_{1}=r_{1}$, then $s_{1}$ is lower bounded iff $r_{1}$ is lower bounded.

(14) If $s_{1}=r_{1}$ and $r_{1}$ is convergent, then $s_{1}$ is convergent to finite number and convergent and $\lim s_{1}=\lim r_{1}$.

(15) If $s_{1}=r_{1}$ and $s_{1}$ is convergent to finite number, then $r_{1}$ is convergent and $\lim s_{1}=\lim r_{1}$.

(16) If $s_{1} \uparrow k$ is convergent to finite number, then $s_{1}$ is convergent to finite number and convergent and $\lim s_{1}=\lim \left(s_{1} \uparrow k\right)$.

(17) If $s_{1} \uparrow k$ is convergent, then $s_{1}$ is convergent and $\lim s_{1}=\lim \left(s_{1} \uparrow k\right)$. 
(18) If $\lim \sup s_{1}=\liminf s_{1}$ and $\liminf s_{1} \in \mathbb{R}$, then there exists $k$ such that $s_{1} \uparrow k$ is bounded.

(19) If $s_{1}$ is convergent to finite number, then there exists $k$ such that $s_{1} \uparrow k$ is bounded.

(20) Suppose $s_{1}$ is convergent to finite number. Then $s_{1} \uparrow k$ is convergent to finite number and $s_{1} \uparrow k$ is convergent and $\lim s_{1}=\lim \left(s_{1} \uparrow k\right)$.

(21) If $s_{1}$ is convergent, then $s_{1} \uparrow k$ is convergent and $\lim s_{1}=\lim \left(s_{1} \uparrow k\right)$.

(22) If $s_{1}$ is upper bounded, then $s_{1} \uparrow k$ is upper bounded and if $s_{1}$ is lower bounded, then $s_{1} \uparrow k$ is lower bounded.

(23) $\quad \inf s_{1} \leq s_{1}(n)$ and $s_{1}(n) \leq \sup s_{1}$.

(24) $\inf s_{1} \leq \sup s_{1}$.

(25) If $s_{1}$ is non-increasing, then $s_{1} \uparrow k$ is non-increasing and $\inf s_{1}=\inf \left(s_{1} \uparrow k\right)$.

(26) If $s_{1}$ is non-decreasing, then $s_{1} \uparrow k$ is non-decreasing and $\sup s_{1}=\sup \left(s_{1} \uparrow\right.$ $k)$.

(27) (The superior realsequence $\left.s_{1}\right)(n)=\sup \left(s_{1} \uparrow n\right)$ and (the inferior realsequence $\left.s_{1}\right)(n)=\inf \left(s_{1} \uparrow n\right)$.

(28) Let $s_{1}$ be a sequence of extended reals and $j$ be an element of $\mathbb{N}$. Then the superior realsequence $s_{1} \uparrow j=\left(\right.$ the superior realsequence $\left.s_{1}\right) \uparrow j$ and $\lim \sup \left(s_{1} \uparrow j\right)=\lim \sup s_{1}$.

(29) Let $s_{1}$ be a sequence of extended reals and $j$ be an element of $\mathbb{N}$. Then the inferior realsequence $s_{1} \uparrow j=$ (the inferior realsequence $\left.s_{1}\right) \uparrow j$ and $\liminf \left(s_{1} \uparrow j\right)=\liminf s_{1}$.

(30) Let $s_{1}$ be a sequence of extended reals and $k$ be an element of $\mathbb{N}$. Suppose $s_{1}$ is non-increasing and $-\infty<s_{1}(k)$ and $s_{1}(k)<+\infty$. Then $s_{1} \uparrow k$ is upper bounded and $\sup \left(s_{1} \uparrow k\right)=s_{1}(k)$.

(31) Let $s_{1}$ be a sequence of extended reals and $k$ be an element of $\mathbb{N}$. Suppose $s_{1}$ is non-decreasing and $-\infty<s_{1}(k)$ and $s_{1}(k)<+\infty$. Then $s_{1} \uparrow k$ is lower bounded and $\inf \left(s_{1} \uparrow k\right)=s_{1}(k)$.

(32) Let $s_{1}$ be a sequence of extended reals. Suppose that for every element $n$ of $\mathbb{N}$ holds $+\infty \leq s_{1}(n)$. Then $s_{1}$ is convergent to $+\infty$.

(33) Let $s_{1}$ be a sequence of extended reals. Suppose that for every element $n$ of $\mathbb{N}$ holds $s_{1}(n) \leq-\infty$. Then $s_{1}$ is convergent to $-\infty$.

(34) Let $s_{1}$ be a sequence of extended reals. Suppose $s_{1}$ is non-increasing and $-\infty=\inf s_{1}$. Then $s_{1}$ is convergent to $-\infty$ and $\lim s_{1}=-\infty$.

(35) Let $s_{1}$ be a sequence of extended reals. Suppose $s_{1}$ is non-decreasing and $+\infty=\sup s_{1}$. Then $s_{1}$ is convergent to $+\infty$ and $\lim s_{1}=+\infty$.

(36) For every sequence $s_{1}$ of extended reals such that $s_{1}$ is non-increasing holds $s_{1}$ is convergent and $\lim s_{1}=\inf s_{1}$. 
(37) For every sequence $s_{1}$ of extended reals such that $s_{1}$ is non-decreasing holds $s_{1}$ is convergent and $\lim s_{1}=\sup s_{1}$.

(38) Let $s_{2}, s_{3}$ be sequences of extended reals. Suppose $s_{2}$ is convergent and $s_{3}$ is convergent and for every element $n$ of $\mathbb{N}$ holds $s_{2}(n) \leq s_{3}(n)$. Then $\lim s_{2} \leq \lim s_{3}$.

(39) For every sequence $s_{1}$ of extended reals holds $\lim \inf s_{1} \leq \lim \sup s_{1}$.

(40) For every sequence $s_{1}$ of extended reals holds $s_{1}$ is convergent iff $\liminf s_{1}=\lim \sup s_{1}$.

(41) For every sequence $s_{1}$ of extended reals such that $s_{1}$ is convergent holds $\lim s_{1}=\liminf s_{1}$ and $\lim s_{1}=\lim \sup s_{1}$.

\section{REFERENCES}

[1] Grzegorz Bancerek. The ordinal numbers. Formalized Mathematics, 1(1):91-96, 1990.

[2] Józef Białas. Infimum and supremum of the set of real numbers. Measure theory. Formalized Mathematics, 2(1):163-171, 1991.

[3] Józef Białas. Series of positive real numbers. Measure theory. Formalized Mathematics, 2(1):173-183, 1991.

[4] Józef Białas. The $\sigma$-additive measure theory. Formalized Mathematics, 2(2):263-270, 1991.

[5] Czesław Byliński. Functions and their basic properties. Formalized Mathematics, 1(1):5565, 1990.

[6] Czesław Byliński. Functions from a set to a set. Formalized Mathematics, 1(1):153-164, 1990.

[7] Czesław Byliński. Partial functions. Formalized Mathematics, 1(2):357-367, 1990.

[8] Czesław Byliński and Piotr Rudnicki. Bounding boxes for compact sets in $\mathcal{E}^{2}$. Formalized Mathematics, 6(3):427-440, 1997.

[9] Noboru Endou and Yasunari Shidama. Integral of measurable function. Formalized Mathematics, 14(2):53-70, 2006.

[10] Noboru Endou, Katsumi Wasaki, and Yasunari Shidama. Definitions and basic properties of measurable functions. Formalized Mathematics, 9(3):495-500, 2001.

[11] Adam Grabowski. On the Kuratowski limit operators. Formalized Mathematics, 11(4):399-409, 2003.

[12] Jarosław Kotowicz. Convergent real sequences. Upper and lower bound of sets of real numbers. Formalized Mathematics, 1(3):477-481, 1990.

[13] Jarosław Kotowicz. Convergent sequences and the limit of sequences. Formalized Mathematics, 1(2):273-275, 1990.

[14] Jarosław Kotowicz. Monotone real sequences. Subsequences. Formalized Mathematics, 1(3):471-475, 1990.

[15] Jarosław Kotowicz. Real sequences and basic operations on them. Formalized Mathematics, 1(2):269-272, 1990.

[16] Andrzej Nȩdzusiak. $\sigma$-fields and probability. Formalized Mathematics, 1(2):401-407, 1990.

[17] Andrzej Trybulec. Subsets of complex numbers. To appear in Formalized Mathematics.

[18] Andrzej Trybulec. Tarski Grothendieck set theory. Formalized Mathematics, 1(1):9-11, 1990.

[19] Zinaida Trybulec. Properties of subsets. Formalized Mathematics, 1(1):67-71, 1990.

[20] Edmund Woronowicz. Relations and their basic properties. Formalized Mathematics, 1(1):73-83, 1990.

[21] Edmund Woronowicz. Relations defined on sets. Formalized Mathematics, 1(1):181-186, 1990.

[22] Bo Zhang, Hiroshi Yamazaki, and Yatsuka Nakamura. Inferior limit and superior limit of sequences of real numbers. Formalized Mathematics, 13(3):375-381, 2005.

Received August 28, 2007 